\title{
The virtues of single atoms
}

\author{
Success in cooling single atoms to millikelvin temperatures, and then of trapping them for \\ seconds (or even hours) on end, will benefit not merely spectroscopists but the rest of physics.
}

THE obvious benefits of being able to study single ions or even atoms are those that the spectroscopists have been extolling for several years, ever since it seemed probable that finely tuned lasers might be used to rob moving atoms of velocity in a specific fashion. Earlier this year, there was a flurry of excitement with the first demonstration that sodium atoms can be "cooled", or robbed of velocity, by the selective absorption of photons (and thus momentum) of a suitable frequency. (The technique and the consequences were fully discussed by B.W. Petley in a News and Views article on 27 June this year, p.716.)

Especially because it is now possible to trap electrically charged particles in suitably configured static electric and magnetic fields, the spectroscopists have had plenty to look for, not least the chance to trap small numbers of ions in excited states so long-lasting that the radiation they emit is neither made fuzzy by the Doppler effect, a consequence of movement, nor broadened substantially by quantum considerations, Heisenberg's uncertainty principle in particular. The notion that suitably prepared trapped atoms may make more convenient standards of frequency (or of time) than the more familiar atomic clocks of caesium is not entirely out of court.

But even before these opportunities have been exploited, the hunt seems well under way for a technique for trapping neutral atoms and molecules in apparently empty space. The first informal report of success in this direction came from Harold Metcalf of the State University of New York at Stony Brook (also in Nature on 27 June, p.717) hinting at experiments by his own group and by another at Bell Laboratories in which neutral sodium atoms had been successfully trapped for periods of time measured in seconds. Now it seems that more systematic ways of achieving these objectives are in sight.

The article by R.V.E. Lovelace, C. Mehanian, T.J. Tommila and D.M. Lee, all of them from various parts of the physics enterprise at Cornell University, is not so much the report of a discovery as a challenge to other experimenters. They explain that the goal they have set themselves theoretically is to define the circumstances in which it would be possible to trap atoms of neutral hydrogen and then to explore the question whether such an assemblage could ever be made dense and cool enough so as to undergo the
Bose-Einstein phase transition expected of all bosons, particles (in this case atoms) with integer spin (zero included).

Will the challenge be taken up? A glance at Table 1 on p. 31 will show how formidable a task lies ahead. At 0.1 degrees above absolute zero (or at $0.1 \mathrm{~K}$ ), it would be necessary to compress hydrogen atoms dealt with singly to roughly the density expected from molecular hydrogen gas at normal temperature and pressure. Doing this in a vacuum will require a substantial magnetic bottle. But how can such a device, familiar enough in the manipulation of some plasmas which are candidates for thermonuclear fuel, be made to function for atoms which have no electric charge? The clever trick that Lovelace et $a l$. describe entails exploiting the potentially different orientations in a magnetic field of the nuclear and electron spins of single hydrogen atoms. It should be possible to trap those atomic arrangements in which both the nuclear and electron spins are parallel to an external magnetic field by exploiting their tendency to congregate at the places where the field is greatest. The snag is that collisions between atoms would require that the experiment would be feasible only at a temperature of 0.0001 $\mathrm{K}$, thought still to be unattainable. Whence the neat proposal that an oscillating magnetic field should be superimposed on a static field in such a way as to create an oscillating magnetic bottle to pump atoms with different hyperfine structure to different regions.

It remains to be seen whether the scheme proposed by Lovelace $\mathrm{et}$ al. can be exploited as they suggest, but the fact that the scheme has arisen at this stage is a proof of how far the physics of single atoms has developed in the past year or so. Whatever may be the defects of this scheme, nobody would have thought it sensible to put it forward five years ago. And it is also plain that the interest of schemes for trapping single atoms and molecules, or small numbers of them, extends far beyond spectroscopy. B.W. Petley explained in June how measurements of stable confined atomic systems could be used to test the isotropy of gravitational inertia. T. Erber and S. Putterman, on p. 41 of this issue, raise another set of questions in fundamental physics that may be answerable by means of atoms and molecules confined in traps. Can such a system be used to test the randomness of the physical processes such as the decay of the

\section{excited state of an atom?}

In principle, it might be possible to carry out such a study by means of a single atom or ion that is suitably trapped in a potential well, using some kind of radiation source to excite it and a suitable photomultiplier to detect the photons arising from radiative decay. But the photons used for excitation would have precisely the same frequency as those to be detected by the photomultiplier. If the average lifetime of the excited atom were to be large enough for the intervals between excitation and decay to be measurable, the excitation would be only weakly coupled to the exciting radiation, which would enhance the likelihood that the photomultiplier would be more often activated by exciting radiation than by that from a genuine decay of an excited atom.

So Erber and Putterman propose a neat atomic switch, a way of telling whether a single atom is at any point excited into one of these comparatively long-lived states. To fit the need, the atom must have two excitation levels, one of which is shortlived and the other long-lived. By means of two lasers, one tuned to each of the excitation frequencies, it should be possible to be sure that the atom at any time is in one of three states, the unexcited ground state or one or other of the two excited states. But the amount of time for which the atom is in the short-lived state can be measured by recording the photons arising by the spontaneous fluorescent decay of that short-lived state. Moreover, because the frequency of the transitions in each direction will be high, if the disparity of lifetimes is great enough, when there is no short-lived fluorescence, the atom should be excited into the long-lived state. In other words, the experiment that naturally suggests itself is a means of measuring the length of time for which a single atom persists in the long-lived state.

Erber and Putterman go on to say that these lifetimes, which should be unaffected by any influence of the external observer, should be strictly random numbers. Paradoxically, they remark, the simplest of all physical systems, a single atom caught in a trap, should be more capable than all but the most complicated computing machinery of generating strictly random numbers. The more important point is that the system is yet another way of testing predictions of quantum mechanics. That is another challenge, but increasingly an academic one.
John Maddox 